\title{
Thérapie cellulaire des maladies musculaires
}

> Les approches de thérapie cellulaire des dystrophinopathies basées sur l'utilisation de myoblastes ou de mésoangioblastes se sont traduites par des résultats cliniques mitigés. De nombreux candidats cellulaires alternatifs ont été décrits, mais aucune comparaison standardisée n'a pu encore établir leurs efficacités, ne serait-ce qu'en vue d'une régénération musculaire localisée. Une étude comparative a donc été décidée récemment et pourrait permettre de donner un nouvel élan à cette approche. <

\section{Problématique de cette voie thérapeutique}

La thérapie cellulaire des dystrophies musculaires est un concept reposant à la fois sur la restauration de l'expression d'une protéine déficiente et la participation à la régénération tissulaire globale. De nombreux candidats cellulaires ont été utilisés chez l'animal selon de nombreuses modalités différentes. Des essais cliniques ont été réalisés, mais ils se sont traduits par des résultats mitigés sur les plans biologiques ou cliniques. La réunion à Paris (juin 2017) d'un panel d'experts très impliqués dans ce domaine (cités dans la bibliographie) a permis d'identifier les leçons à tirer des échecs et d'envisager une stratégie permettant de redessiner l'avenir de cette approche.

\section{Pourquoi persévérer?}

À l'heure actuelle, la thérapie cellulaire est considérée comme un traitement palliatif potentiel, mais non curatif au plan systémique, car limité à certaines zones d'injection dans le corps. Les approches de thérapie génique sont de plus en plus nombreuses, élaborées, et fructueuses, mais thérapies géniques et cellulaires sont par essence complémentaires parce que les capacités de régénération d'un muscle déjà dystrophique - même après «réparation » génique - sont a priori trop limitées pour permettre un gain fonctionnel utile. Ceci est particulièrement avéré pour les petits muscles fonctionnellement nécessaires à

\section{Un avenir à l'aune} d'une comparaison des progéniteurs

\section{Jean-Thomas Vilquin ${ }^{1}$, Serge Braun ${ }^{2}$}

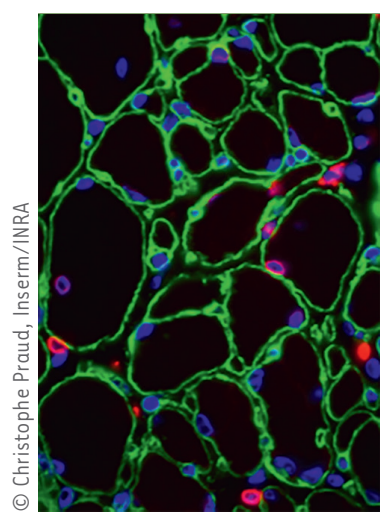

${ }^{1}$ Sorbonne Université, Inserm UMRS 974, AIM, CNRS, Centre de Recherche en Myologie, Hôpital Pitié Salpêtrière, Faculté de Médecine, 105 boulevard de I'Hôpital, 75013 Paris, France. ${ }^{2}$ AFM-Téléthon, 1 rue de l'Internationale, 91000 Évry, France. Lean-Thomas.Vilquin@institutmyologie.org

l'autonomie des personnes (muscles de la main). Localement, cette approche pourrait toutefois permettre de préserver ou d'améliorer la qualité de vie des patients; le caractère invasif temporaire de cette procédure n'invalide pas son utilité thérapeutique finale.

Beaucoup de questions sont encore en suspens, leur résolution pourrait permettre d'améliorer grandement le succès de ces greffes: combien de cellules sont nécessaires pour parvenir à la régénération? Quels sont les meilleurs paramètres d'injection puis de quantification? Peut-on pallier des problèmes biologiques (mort cellulaire, migration...) par des procédures techniques?

Les candidats cellulaires sont nombreux et une multitude d'études précliniques et même cliniques ont été menées, mais aucune étude standardisée n'a encore été réalisée pour comparer leurs efficacités relatives, bien que certaines études individuelles aient été très poussées [1]. II faut souligner que certaines limitations sont intrinsèques à chaque type cellulaire (capacité de fusion, de migration, résistance au stress oxydant et à la mort cellulaire immédiate...) et pourraient constituer des facteurs de sélection parmi ces candidats, tandis que d'autres limitations sont plus globales (rejet immunologique) ou extrinsèques (âge des patients donneurs ou receveurs) et nécessiteraient des mesures d'ordre général. Certaines limitations importantes telles que la mort cellulaire ont été très peu étudiées, en dehors du cas des myoblastes, et surtout chez la souris.

S'il est donc trop tôt pour conclure à l'inefficacité générale de la thérapie cellulaire dans les maladies neuromusculaires, il est encore 
temps de procéder à une étude comparative et systématique, en réunissant les différents outils mis à notre disposition par des années d'expérimentations.

\section{Quels candidats cellulaires, pour quel modèle animal ?}

Dans une perspective de développement clinique ultérieur, seuls les types cellulaires d'origine humaine seront comparés.

Les cellules satellites constituent la principale réserve myogénique mais elles perdent leur statut en culture, lorsqu'elles sont activées et deviennent des progéniteurs, les myoblastes [2, 3]. Testés à de nombreuses reprises, ces myoblastes constituent un standard [4] mais leur efficacité est limitée par une faible capacité migratoire tissulaire [5], bien qu'ils puissent persister durant des années au sein du tissu hôte [6]. Utilisés en injections multisites, ils permettent seulement une restauration locale d'expression de la dystrophine dans les modèles de dystrophinopathies $[7,8]$.

Les péricytes, les mésoangioblastes et les cellules adventitielles font plutôt partie des cellules angiogéniques et/ou de soutien mais ont également une capacité myogénique importante $[9,10]$. Les mésoangioblastes ont été testés par injection systémique, mais n'ont pas permis de restauration significative de la dystrophine, et bien que ces cellules se soient montrées très efficaces dans le modèle canin de la dystrophie musculaire de Duchenne (DMD) [11].

Les cellules Muscle-Derived Stem Cell (MDSC) et Mustem constituent de petites populations de cellules rares mais à multipotence élevée et présentant un potentiel immunomodulateur. Les cellules MDSC sont utilisées en essais cliniques pour le traitement de l'insuffisance urinaire sphinctérienne, en injection localisée [12]. Les cellules Mustem améliorent le statut clinique des chiens modèles de la DMD après injection systémique [13].

Les cellules CD133 + partageraient des caractéristiques hématopoïétiques et peuvent être extraites des tissus humains, et injectées dans les muscles dystrophiques [14].

Les cellules ALDH+ constituent de petites populations à haute capacité myogénique et particulièrement résistantes au stress oxydant $[15,16]$. Les cellules stromales mésenchymateuses d'origine adipeuse ne proviennent pas du muscle mais ont des capacités paracrines et immunomodulatrices particulièrement importantes.

À côté des cellules adultes ont émergé les cellules pluripotentes, d'origine embryonnaire (cellules ES) ou somatique (cellules iPS). Il est possible à présent d'obtenir leur différenciation myogénique, en passant par différents états d'engagements successifs, à l'aide de combinaisons de facteurs pharmacologiques exogènes et compatibles avec un usage clinique [17]. On peut ainsi comparer des types cellulaires obtenus par différents protocoles, ou à différents états d'engagement. Les cellules iPS provenant de patients pourraient également être modifiées génétiquement ex vivo pour devenir des outils de thérapie cellulaire à usage autologue, particulièrement dans des indications de maladies rares $[18,19]$.

La transplantation de cellules humaines chez l'animal est une xénogreffe, qui doit donc être réalisée dans un contexte immunodéficient (souris SCID). Pour se rapprocher au moins sur le plan biochimique de la maladie humaine, le modèle de souris $m d x$ sera choisi, sa petite taille permettant la réalisation du criblage de l'efficacité de tous les types cellulaires. La première étude sera réalisée par voie intramusculaire directe.

\section{Des outils d'évaluation classiques et standardisés}

L'objectif principal du projet est de comparer l'efficacité de progéniteurs myogéniques pour leurs capacités de régénération dans un modèle animal immunodéficient de DMD.

Les différents types de progéniteurs seront générés directement ou indirectement par les participants au projet, à partir de biopsies musculaires communes fournies par Myobank (la banque de cellules et tissus de l'AFMTéléthon), puis les cellules seront injectées localement, d'une manière standardisée, au niveau d'un muscle unique. A différents intervalles, les muscles seront prélevés et analysés pour étudier et comparer les étendues de régénérations, là encore de manière standardisée.

La présence des cellules humaines au sein du tissu de l'hôte, participant directement à la formation des fibres musculaires squelettiques et/ou demeurant en position endomysiale ou endothéliale, peut être très finement caractérisée par des méthodes immunohistologiques et d'imagerie quantitative standardisées, ce qui permet de suivre le devenir biologique de ces différentes catégories de progéniteurs. La proportion de cellules provenant du donneur et son évolution au cours du temps est quantifiable par PCR, renseignant sur les processus de mort cellulaire immédiate, et de prolifération. L'expression de la dystrophine, et/ou de protéines restaurées, peut être mesurée par Western blot.

Pris ensemble, ces outils permettront un classement multiparamétrique de l'efficacité des différentes populations, et le choix de quelques-unes pour la poursuite de la comparaison, en faisant appel le cas échéant à des modèles animaux de plus grande taille (Figure 1).

\section{Les études complémentaires et les perspectives}

La mise en place de ce protocole fournit l'opportunité de comparer directement, outre leurs capacités fonctionnelles, les caractéristiques phénotypiques et biologiques des différentes catégories de progéniteurs. Cette étude permettra aussi de définir de nouveaux marqueurs ou de nouvelles combinaisons qui seront utiles pour la validation des cellules auprès des agences réglementaires. 


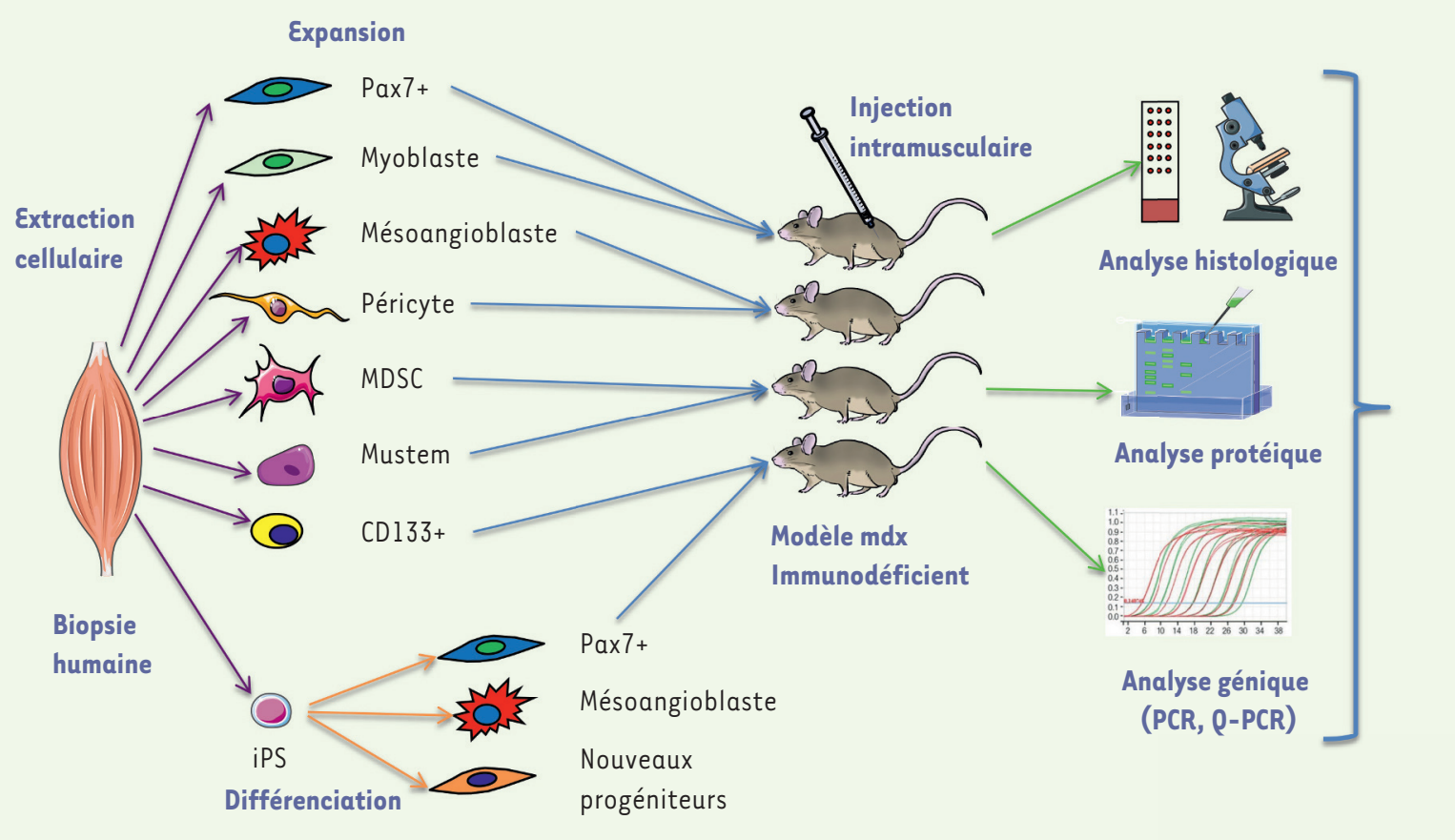

Figure 1. Schéma de l'étude comparative des progéniteurs myogéniques. À partir de biopsies musculaires humaines, les différentes catégories de cellules seront extraites et amplifiées. En parallèle, des cellules pluripotentes seront générées puis différenciées selon différents protocoles. Les cellules seront injectées dans les muscles de souris $\mathrm{mdx}$ immunodéficientes, leur devenir et les résultats d'implantation seront comparés par des techniques complémentaires standardisées.

Une composante importante de la DMD réside dans ses aspects inflammatoires liés aux processus de dégénérescence et régénération. Une étude de l'inflammasome et de sa modulation par les différentes catégories de progéniteurs participera aussi à la compréhension du mécanisme d'action de ces cellules.

Après avoir cerné les meilleurs candidats, des études fonctionnelles seront réalisées sur de nouvelles cohortes d'animaux en restreignant le nombre de progéniteurs testés, afin de confirmer les capacités régénératrices identifiées précédemment. Les fonctions motrices (force, résistance à la fatigue et à l'exercice excentrique) seront mesurées à moyen et long terme sur les muscles greffés.

L'injection intramusculaire est forcément limitée à une étendue thérapeutique locale. Peut-être son efficacité pourrait-elle être améliorée par l'association à des biomatériels. Cependant, certains territoires musculaires ne peuvent être atteints aisément (diaphragme, muscles intercostaux...). La distribution régionale ou systémique par voie vasculaire permettrait de toucher un grand nombre de territoires musculaires simultanément et sans avoir à recourir à des injections ponctuelles multiples. Cependant, nous ignorons quels types cellulaires seraient capables de franchir des barrières vasculaires. II est nécessaire de développer ces approches, au besoin en empruntant le cheminement global qui aura été établi pour les études intramusculaires, afin là encore d'identifier le ou les meilleurs candidats de thérapie cellulaire. Une étude de biodistribution et de tolérance complètera les carac- téristiques fonctionnelles et thérapeutiques des meilleurs candidats. $\diamond$

\section{SUMMARY}

Cell therapy in muscular disorders: the future lies in comparisons of progenitors

Cell therapy approaches dedicated to the treatment of dystrophinopathies and involving essentially myoblasts and mesoangioblasts have produced mitigated clinical results. If several types of alternative progenitors have been developed, no standardized comparison has been carried out yet to investigate their regenerative efficacy in vivo, at least at a local level. A comparative study has therefore been designed recently aiming at giving a new impetus to this therapeutic field. $\diamond$

\section{LIENS D'INTÉRÊT}

Les auteurs déclarent n'avoir aucun lien d'intérêt concernant les données publiées dans cet article.

\section{RÉFÉRENCES}

1. Skuk D, Goulet M, Tremblay JP. Intramuscular transplantation of myogenic cells in primates: importance of needle size, cell number, and injection volume. Cell Transplant $2014 ; 23: 13-25$.

2. Montarras D, Morgan J, Collins C, et al. Direct isolation of satellite cells for skeletal muscle regeneration. Science 2005 ; 309 : 2064-7. 


\section{RÉFÉRENCES}

3. Collins CA, Olsen I, Zammit PS, et al. Stem cell function, self-renewal, and behavioral heterogeneity of cells from the adult muscle satellite cell niche. Cell $2005 ; 122: 289-301$.

4. Alameddine HS, Dehaupas M, Fardeau M. Regeneration of skeletal muscle fibers from autologous satellite cells multiplied in vitro. An experimental model for testing cultured cell myogenicity. Muscle Nerve $1989 ; 12: 544-55$.

5. Skuk D, Goulet M, Tremblay JP. Transplanted myoblasts can migrate several millimeters to fuse with damaged myofibers in nonhuman primate skeletal muscle. J Neuropathol Exp Neurol 2011 $70: 770-8$.

6. Crahès $M$, Bories $M C$, Viquin JT, et al. Long-term engraftment ( 16 years) of myoblasts in a human infarcted heart. Stem Cells Transl Med 2018 ; 7 : 705-8.

7. Tremblay JP, Malouin F, Roy R, et al. Results of a triple blind clinical study of myoblast transplantations without immunosuppressive treatment in young boys with Duchenne muscula dystrophy. Cell Transplant $1993 ; 2: 99-112$.

8. Skuk D, Goulet M, Roy B, et al. Dystrophin expression in muscles of Duchenne muscular dystrophy patients after high-density injections of normal myogenic cells. J Neuropathol Exp Neurol 2006 ; $65: 371-86$.

9. Dellavalle A, Sampaolesi M, Tonlorenzi R, et al. Pericytes of human skeletal muscle are myogenic precursors distinct from satellite cells. Nat Cell Biol $2007 ; 9: 255-67$.

10. Crisan M, Yap S, Casteilla L, et al. A perivascular origin for mesenchymal stem cells in multiple human organs. Cell Stem Cell $2008 ; 3: 301-13$.

11. Cossu G, Previtali SC, Napolitano S, et al. Intra-arterial transplantation of HLA-matched donor mesoangioblasts in Duchenne muscular dystrophy. EMBO Mol Med 2015 ; 7 : 1513-28.

12. Qu-Petersen $Z$, Deasy $B$, Jankowski $R$, et al. Identification of a novel population of muscle stem cells in mice: potential for muscle regeneration. J Cell Biol 2002 ; 157 : 851-64.
13. Rouger K, Larcher T, Dubreil L, et al. Systemic delivery of allogenic muscle stem cells induces long-term muscle repair and clinical efficacy in Duchenne muscular dystrophy dogs. Am J Pathol 2011; 179 : 2501-18.

14. Torrente $Y$, Belicchi M, Marchesi C, et al. Autologous transplantation of muscle-derived CD133 stem cells in Duchenne muscle patients. Cell Transplant $2007 ; 16: 563-77$

15. Vauchez K, Marolleau JP, Schmid M, et al. Aldehyde dehydrogenase activity identifies a population of human skeletal muscle cells with high myogenic capacities. Mol Ther $2009 ; 17: 1948-58$.

16. Jean $\varepsilon$, Laoudj-Chenivesse $D$, Notarnicola C, et al. Aldehyde dehydrogenase activity promotes survival of human muscle precursor cells. J Cell Mol Med $2011 ; 15: 119-33$

17. Chal J, Al Tanoury Z, Hestin M, et al. Generation of human muscle fibers and satellite-like cells from human pluripotent stem cells in vitro. Nat Protoc $2016 ; 11: 1833-50$.

18. Tedesco FS, Gerli MF, Perani L, et al. Transplantation of genetically corrected human iPSC-derived progenitors in mice with limb-girdle muscular dystrophy. Sci Transl Med $2012 ; 4$ : 140ra89.

19. Quattrocelli M, Swinnen M, Giacomazzi G, et al. Mesodermal iPSC-derived progenitor cells functionally regenerate cardiac and skeletal muscle. J Clin Invest 2015 ; 125 : 4463-82.

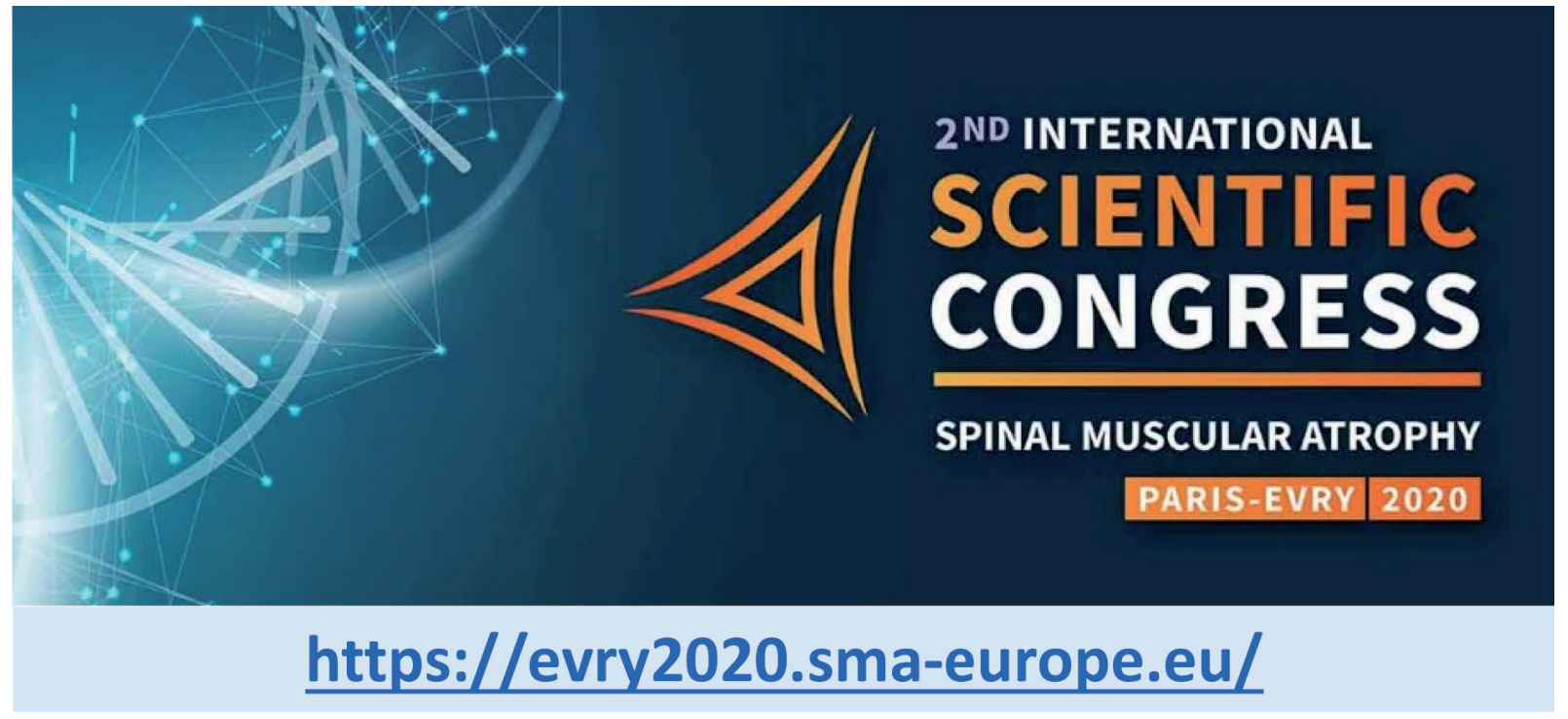

\section{Retrouvez toutes les Actualités de la Myologie sur les sites de :}

TIRÉS À PART

J.T. Vilquin 